\title{
Line Sensor for Fast, Time-Resolved Spectroscopy Measurements
}

\author{
E.Poklonskaya, D.Durini, M.Jung, O.Schrey, W.Brockherde
}

Fraunhofer IMS, Finkenstr.61,

Duisburg, Germany,

elena.poklonskaya@ims.fraunhofer.de

\begin{abstract}
:
In this work a novel CMOS line sensor based on the lateral drift-field photodetector (LDPD) [1] pixel approach is presented. The proposed pixel structure features the photoactive area of $200 \mu \mathrm{m} \times 10 \mu \mathrm{m}$ fulfilling the application requirements [2]. A specially designed $n$-well within the LDPD provides the fast and efficient transfer of the generated carries from the photoactive area into each of the sense or drain nodes. The grounded $p+$ layer localized on the surface of the LDPD n-well reduces the dark current generated by Schockley-Read-Hall recombination/generation centers located on the silicon surface. The UV-enhanced silicon-nitride based passivation layer guarantees the photodiode (PD) sensitivity down to $220 \mathrm{~nm}$ wavelength range. The presented line sensor LDPD pixel is to be employed in optical emission spectroscopy applications.
\end{abstract}

Key words: CMOS Line sensor, LDPD (Lateral Drift Photodiode), Time-Resolved Spectroscopy

\section{Introduction}

Many industrial applications require linear photosensors which exhibit high sensitivity and low noise. The atomic emission spectroscopy (spark spectrometry) is one of those. Such spectroscopic method delivers the information about the qualitative and quantitative composition of an analyte [3]. Since 1960 photomultiplier tubes (PMT) were used as standard detectors in the field of spark spectrometry due to their high speed of response and low dark current. Being sensitive only to a certain impinging radiation photomultipliers require a lot of time to record the entire targeted wavelength spectrum.

Recently, solid-state line sensors have established themselves as a promising alternative to photomultiplier tubes. Providing the possibility of detecting emission lines between $250 \mathrm{~nm}$ and $1 \mu \mathrm{m}$, the developed CMOS photodetectors have the advantage of recording the complete emission spectrum each time.

In spark emission spectroscopy application, numeric atomic and ionic lines are excited and emitted during the spark plasma discharge. However, only a certain amount of these lines contains the information about the desired element. The outcoming spectrum will also hold interfering lines or a high level of continuous background radiation [3]. Typically the emission of ionic nondesired lines occurs earlier than atomic lines. Thus, with the help of time-resolved spectroscopy measurements many atomic lines can be efficiently detected. This method should be able to define a right time window for the collection of the photogenerated charges belonging to each emission channel (gate integration) [3]. The possibility of the so called time gating together with the feature of non-destructive readout and charge accumulation over several cycles without the need of resetting the experiment are the major advantages of the designed and 
implemented LDPD based CMOS line sensor we are presenting in this paper.

\section{Developed Lateral Drift Field Photodector}

Summarizing all the above, the photodetector requirements for spark emission spectroscopy applications are: high sensitivity in a wavelength range between $250 \mathrm{~nm}$ and $1 \mu \mathrm{m}$, low noise, specifically designed pixel area (pixel width of about $10 \mu \mathrm{m}$ and a height up to $1 \mathrm{~mm}$ ), time-resolved measurements, nondestructive readout and accumulation of the signal charges in the sense node over several measurements cycles.

The proposed CMOS line sensor based on the developed lateral drift-field photodetector (LDPD) pixel fulfills these conditions. The pinned area [2] of the pixel consists of an extra n-well incorporating a non-uniform lateral doping profile (Fig.1). It remains fully depleted during the operation, if sandwiched between the substrate and a grounded $p+$ layer, localized on the surface of this $n$-well. The induced concentration gradient within the $n$ well in the direction of the readout node and the unpinned region of the detector generates an electrostatic potential i.e., lateral drift-field [1], which enables not only a better charge collection within the considerably extend photoactive area $(200 \mu \mathrm{m})$, but also a high speed of the charge transfer from this photoactive area to the readout nodes.

A collection-gate (CG), two transfer-gates (TG and $D G$ ), a floating diffusion (FD) and an $n_{+}$ diffusion directly connected to a higher potential and thus called draining diffusion (DD) (Fig.1) all form part of the unpinned area of the deployed LDPD. The CG creates a region of constant electrostatic potential within the LDPD n-well beneath it, and assures equal probabilities for charge transfer from this region to any of the sense nodes placed aside it.

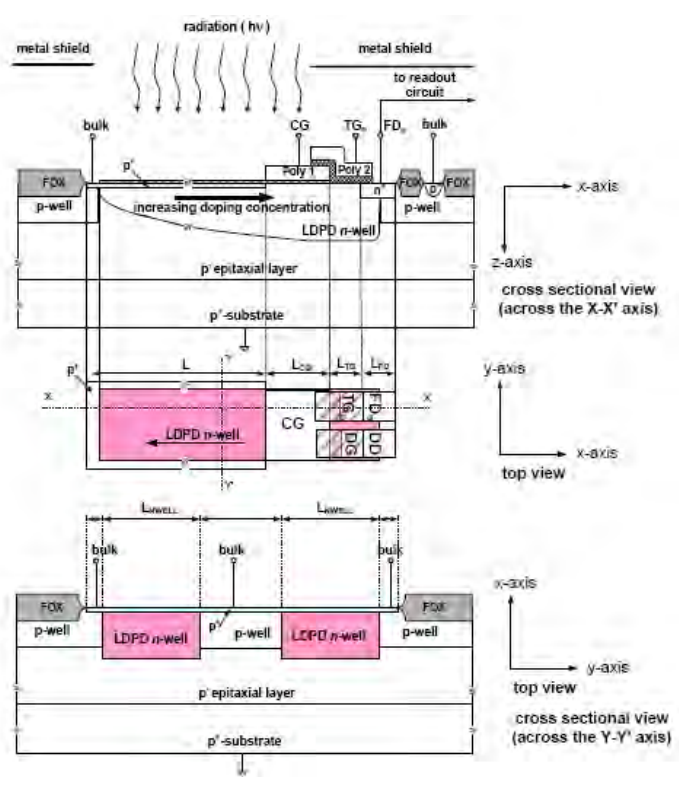

Fig.1. Cross-sectional schematic view of the lateral drift-field photodetector (LDPD).

Correctly biasing the TG, a potential barrier can be created within the well, thus preventing the charge carriers to be transferred to the FD during the charge readout cycle. On the other hand, during the charge collection cycle, the properly biased TG enhances the lateral-drift field mechanism and supports charge carrier transfer.

The DG serves to prevent the electrons being drained out to $V_{d d}$ during the collection cycle of the desired photogenerated charges. And serves to drain the non-desired charge out of the pixel when necessary, providing the system with the time-controlled charge collection ability [4].

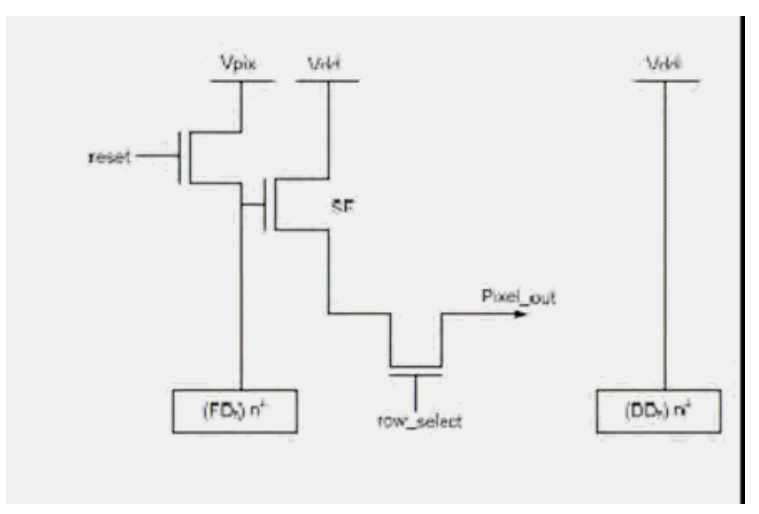

Fig.2. Schematic of the in-pixel readout circuit for both the floating diffusions (FDn) and the drain diffusion (DD).

Fig.2 shows the in-pixel readout circuits, where the FD is connected to the source electrode of a "reset transistor", from which the signal can be read out through an in-pixel buffer built in a source-follower configuration [2], while the DD 
is directly connected to a higher potential, here depicted as VDD.

Due to the special UV-enhanced silicon-nitride based passivation layer, the LDPD structure provides good blue and UV quantum efficiency down to $220 \mathrm{~nm}$. A comparison of the quantum efficiency and optical sensitivity curves of the LDPD-pixels using the standard passivation, normally present in the CMOS process, and the UV-enhanced, specially developed for this application, one can be observed in Fig. 3 .

The UV-enhanced silicon nitride based passivation layer contains a higher amount of nitrogen compared to the standard passivation. Thus, the extinction coefficient for lower wavelengths is significantly reduced yielding higher transmittance in the UV range of the spectrum.

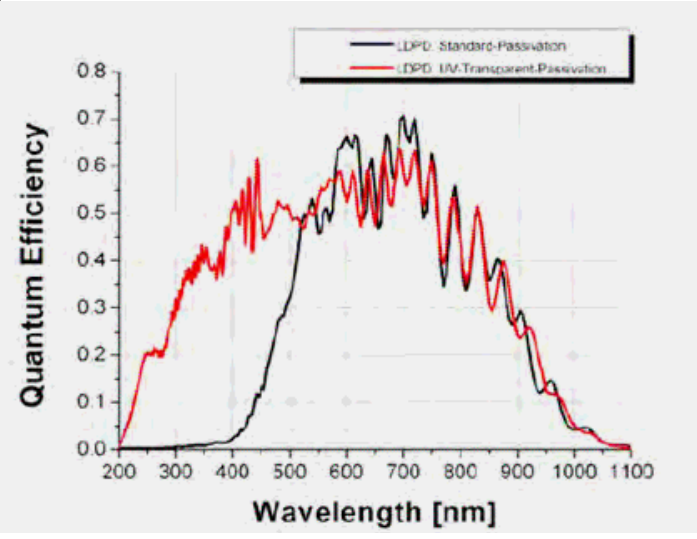

Fig.3. Wavelength dependent quantum efficiency curves obtained from the LDPD-line sensors using two different types of passivation layers.

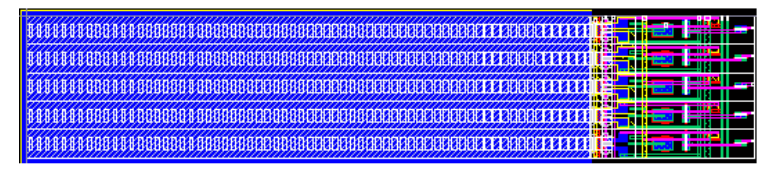

Fig.4. Layout used for the LDPD test structures (5 pixels).

\section{Test array}

A test array with several 5-pixel test clusters and an output buffer was fabricated using the $0.35 \mu \mathrm{m} \mathrm{LV/HV} \mathrm{CMOS} \mathrm{process} \mathrm{with} \mathrm{an} \mathrm{LDPD}$ and a specially designed UV-enhanced siliconnitride based passivation. The actual layout used for the fabrication of the 5-pixel test fields can be observed in Fig. 4. Consistent with Fig.1, here $L=200 \mu \mathrm{m}$ is the length of the pixel photoactive area. The distance between the neighboring pixels $\mathrm{n}$-well is $5.5 \mu \mathrm{m}$ for a $10 \mu \mathrm{m}$ pixel pitch.

The dark current measured is of $320 \mathrm{pA} / \mathrm{cm}^{2}$, referred to the entire pixel area. The full-well capacity (FWC) of the pixel is of $190.000 \mathrm{e}$-, for a reached sensitivity of $6 \mu \mathrm{V} / \mathrm{e}$ - and the responsivity of $165 \mathrm{~V} / \mu \mathrm{J} / \mathrm{cm}^{2}$ for $\lambda=525 \mathrm{~nm}$ of impinging radiation. The obtained dark current is too high for the proposed application and the sensitivity is too low, so the further optimization of the LDPD pixel has to be done.

Therefore, new LDPD test structures with $\mathrm{L}=200 \mu \mathrm{m}$ and $10 \mu \mathrm{m}$ pitch, organized with the same principle of having 19 different 5-pixels clusters were proposed. The newly optimized LDPD-pixel structure was simulated using the process and device simulation tools (Synopsys TCAD), fabricated in the same $0.35 \mu \mathrm{m} \mathrm{LV/HV}$ CMOS process and characterized by means of the Photon Transfer Method (PTM). The obtained results are discussed below.

\section{Simulation}

Having such a long photoactive area due to the application requirements, it is considered difficult to realize the desired potential gradient within the n-well without performing necessary calculation and a TCAD simulation.

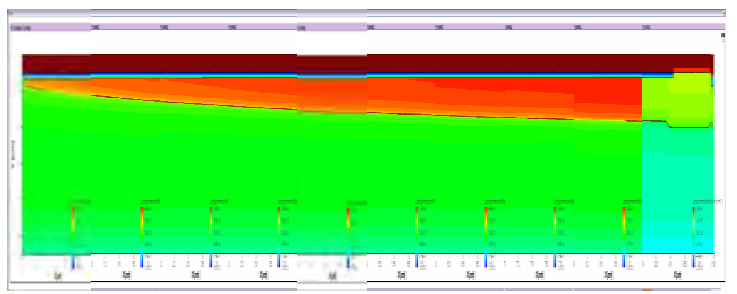

Fig.5. 2D TCAD simulation of the LDPD n-well doping profile.

The exact calculation of an extra n-well mask for creating nonuniformal doping concentration profile which lead to the generation of the electrostatic potential gradient within the $n$-well described in [1] .TCAD simulation of the n-well clearly exhibits the desired doping profile on the entire length of the photoactive area (Fig 5.)

Another very important issue which has to be taken into consideration by fabricating the proposed CMOS line sensor is how to separate neighboring pixels in order to avoid crosstalk among their diffused n-wells. To investigate this potential problematic, the interface between two photoactive areas was simulated. The simulation results are depicted on Fig. 6. and confirmed that both photoactive areas are perfectly separated.

A similar problem arises in the area between the TG and DG. However, the simulated electrostatic potential profile on Fig. 7 shows that the $p$-well located between the two n-wells under the gates induces a potential barrier, which prevents the charge carrier crosstalk between the TG and DG. 
Even putting the gates closer to each other the desired potential barrier remains and servers its purpose.

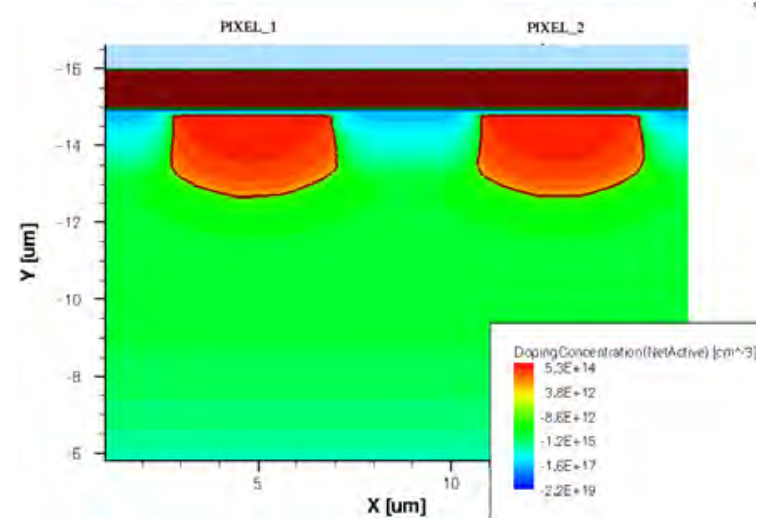

Fig.6. 2D TCAD simulation of the n-well of two neighboring $L D P D$ pixels.

\section{Measurement Results}

The developed LDPDs were characterized by means of the PTM. After a series of optimizations the LDPD yielded $96 \mathrm{pA} / \mathrm{cm}^{2}$ of dark current at room temperature defined to the entire pixel area, $50.000 \mathrm{e}^{-}$of FWC, a responsivity of $450 \mathrm{~V} / \mu \mathrm{J} / \mathrm{cm}^{2}$ for $\lambda=525 \mathrm{~nm}$ of impinging radiation, and a sensitivity of $17 \mu \mathrm{V} / \mathrm{e}^{-}$. All additional parameters specified and measured are presented in Table 1.

The responsivity was increased by accurate designing of the n-well and a proper choice of the recipe for the pinning layer implantation in order to achieve the desired horizontal doping gradient in the $n$-well and increase the depletion region.

The transfer time is a major concern during the development of a CMOS line sensor due to the extra long photoactive area, which introduces difficulties in the collection and transport of the charge carriers. The increase of the electrostatic potential gradient and correctly biased $C G$ and $T G$ make the transfer of the charge carriers fast and efficient. Moreover, by changing the geometry of the FD and SF transistor the capacitance of the sense node can be adjusted.

The atomic emission spectroscopy requires low PD dark currents. Introducing the additional $\mathrm{p}+$ layer on top of the silicon surface, the recombination mechanism on the silicon surface is supported and thus the number of charge carriers generated thermally from the surface states and collected within the photodetector can be reduced [5]. Another source of the generated dark current are the Schockley-Read-Hall recombination/generation centers located on the silicon surface under the $C G$ and TG. Thus the electrostatic potential maximum should be put away from the $\mathrm{Si} / \mathrm{SiO}_{2}$ interface, in order to decrease this source of the dark current.

The developed LDPD after optimization still shows considerably high dark current, which needs to be optimized in the future.

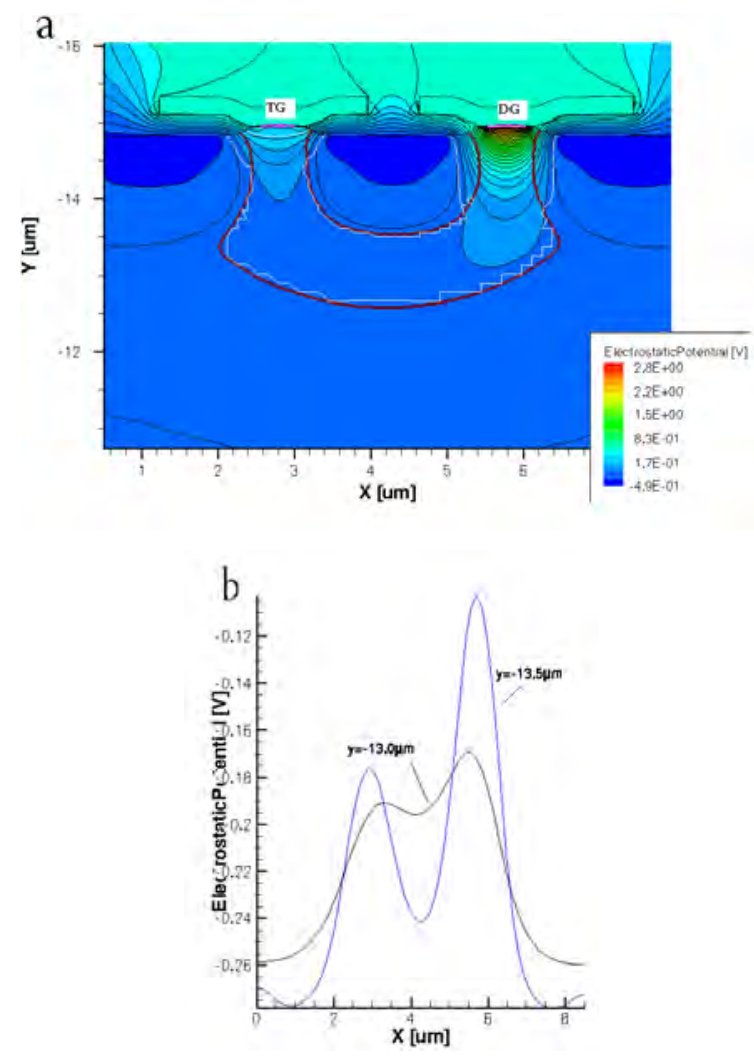

Fig.6 (a) $2 D$ TCAD simulation of the one pixel electrostatic potential between $T G$ and $D G$, the potential on the $n$-well under the TG is OV, under the $D G 2.5 \mathrm{~V}$ (b) electrostatic potential under the TG and DG for two different distances between the gates.

\section{Conclusion}

Within the frame of this work, an LDPD line sensor has been developed which can be used for time resolved measurements. The designed detector is sensitive in the UV part of the spectrum and exhibits an acceptable sensitivity for the application requirements. High DR (Dynamic Range), achieved, among other parameters, also through the accumulation of the signal charge over several measurement cycles without the need of resetting the sense node(and so introducing the kTC noise after each cycle) and low dark current make the proposed LDPD an ideal candidate for atomic emission spectroscopy applications.

Tab.1 Electro-optical parameters of the developed $\angle D P D$ pixel with the length of the photoactive area 200 um verified by means of PTM. The integration time is $t_{I N T}=60 \mu$ s while the sensor was illuminated 
with monochromatic radiation at $\lambda=525 \mathrm{~nm}$ and $80000 \mathrm{nW} / \mathrm{cm}^{2}$ illumination intensity.

\begin{tabular}{|c|c|c|}
\hline Specifications & Units & Result \\
\hline $\begin{array}{c}\text { Responsivity } \\
(\mathrm{R})\end{array}$ & {$\left[\mathrm{V} / \mu \mathrm{J} / \mathrm{cm}^{2}\right]$} & 451 \\
\hline $\begin{array}{c}\text { Saturation } \\
\text { Capacity } \\
(\mathrm{SC})\end{array}$ & {$[\mathrm{ke}]$} & 50 \\
\hline $\begin{array}{c}\text { Sensitivity } \\
(\mathrm{S})\end{array}$ & {$\left[\mu \mathrm{V} / \mathrm{e}^{\mathrm{e}}\right]$} & 17,4 \\
\hline $\begin{array}{c}\text { Signal -to }- \\
\text { Noise Ratio } \\
(\mathrm{SNR})\end{array}$ & {$[\mathrm{dB}]$} & 48,2 \\
\hline $\begin{array}{c}\text { Dynamic } \\
\text { Range } \\
(\mathrm{DR})\end{array}$ & {$[\mathrm{dB}]$} & 58,5 \\
\hline $\begin{array}{c}\text { Dark Current } \\
\mathrm{T}=25-26^{\circ} \mathrm{C} \\
\mathrm{t}_{\mathrm{NNT}}=0-20 \mathrm{~s}\end{array}$ & $\mathrm{pA} / \mathrm{cm}^{2}$ & 96 \\
\hline
\end{tabular}

[5] D. Durini, W. Brockherde, and B. J. Hosticka. "Detector" U.S. Patent 2010/0308213 A1, Dec.9, 2010.

\section{References}

[1] D. Durini, A. Spickermann, R. Mahdi, W. Brockherde, H. Vogt, A. Grabmaier, and B. J. Hosticka,"Lateral drift-field photodiode for lownoise, high-speed, large photoactive-area CMOS imaging applications," Nuclear Instruments and Methods in Physics Research A 624, pp. 470-475, 2010

[2] A. J. P. Theuwissen, "CMOS Image Sensors: State-of-the-Art", Solid-State Electronics 52, pp. 1401-1406, 2008

[3] Volker B.E. Thomsen, "Modern Spectrochemical Analysis of Metals", ASM International, 1996

[4] R.Mahdi, J.Fink, and B. J. Hosticka," Lateral Drift-Field Photodetector for High Speed 0.35 $\mu \mathrm{m}$ CMOS Imaging Sensors Based on Non-Uniform Lateral Doping Profile," Ph.D. Research in Microelectronics and Electronics (PRIME), 2010 Conference on , vol., no., pp.1-4, 18-21 July 2010 